\title{
Metronidazole Hypersensitivity in a Patient With Angioedema and Widespread Rash
}

Aruanno A*, Parrinello G*, Buonomo A, Rizzi A, Nucera E Allergy Unit, Fondazione Policlinico Universitario A. Gemelli IRCCS, Rome, Italy

*Both authors contributed equally to this work.

J Investig Allergol Clin Immunol 2020; Vol. 30(5): 371-373 doi: 10.18176/jiaci.0554

Key words: Metronidazole. Hypersensitivity. Delayed systemic reaction.

Palabras clave: Metronidazol. Hipersensibilidad. Reacción sistémica retardada.

Metronidazole is a 5-nitroimidazole compound that was introduced in 1959 to treat Trichomonas vaginalis infections. It shares a high structural similarity with its derivatives (tinidazole, secnidazole, and ornidazole) and is used to treat parasitic infections, alone or in combination with other antibiotics. The drug is usually well tolerated, but may occasionally cause adverse effects (gastrointestinal symptoms, hematological alterations, central nervous system disorders, and, rarely, drug rashes) $[1,2]$.

We can also find metronidazole in face creams and cosmetics. Sensitization may occur after their topical application.

We report a case of labial angioedema and widespread erythematous rash in a patient with type IV allergy to metronidazole.

A 45-year-old nonatopic man developed labial angioedema and widespread itchy erythematous maculopapular rash some 10 hours after the third dose of oral metronidazole prescribed for gastrointestinal dysbiosis. Symptoms disappeared within a few hours of administration of intravenous chlorphenamine and methylprednisolone. At the time of the event, the patient's blood count was normal, with no eosinophilia or lymphocytosis; there was no organ involvement, inflammatory markers were normal, and the patient was afebrile. After the event, he avoided the use of metronidazole in any form.

Three years later, the patient was admitted to the Allergy Department of Fondazione Policlinico Universitario A. Gemelli IRCCS, Rome, Italy for an allergological work-up, which included skin prick tests (SPTs) and patch tests with metronidazole, as reported elsewhere [1,2]. The SPTs were carried out with metronidazole on the volar area of the forearm at $125 \mathrm{mg} / \mathrm{mL}$ in the form of powered tablets dissolved in saline. Histamine $(10 \mathrm{mg} / \mathrm{mL})$ was used as a positive control and saline $(0.9 \%)$ as a negative control. Patch tests were applied on the interscapular region and carried out with metronidazole in the form of powdered tablets dissolved in petrolatum at concentrations of $0.5 \%, 5 \%$, and $10 \%$ and with the undiluted solution. We also performed both tests in 10 healthy controls.

The SPT result was negative, while the patch tests indicated a positive reaction after 72 hours, with an erythematous 
infiltrate of $12 \mathrm{~mm}$ for the undiluted solution. The results were negative in all the healthy controls. The same allergological work-up performed with other imidazole derivatives (tinidazole, albendazole, mebendazole, tioconazole) yielded negative results.

The patient refused to undergo an oral provocation test (OPT). However, his clinical history and allergy testing results were highly suggestive of cell-mediated allergy (type IV reaction) to metronidazole. We recommended future avoidance of this drug and the other imidazole derivatives.

Hypersensitivity reactions to metronidazole are rare. However, they are becoming more frequent because the drug is increasingly used in combination with other antibiotics to treat amebiasis and anaerobic infections.

The various reported cases of hypersensitivity reactions to metronidazole have taken the form of cutaneous adverse reactions (eg, allergic contact dermatitis) [4], fixed drug eruptions [5], systemic reactions [1-3], respiratory disorders [6,7], anaphylactic reactions [2], StevensJohnson syndrome/toxic epidermal necrolysis [8], acute generalized exanthematous pustulosis [9], and serum sickness reactions [10].

Most of the systemic reactions described were not confirmed by allergy testing, except for some cases of immediate reactions confirmed by a positive SPT result $[1,2]$. Instead, most reactions to this drug confirmed by positive patch test results were essentially allergic contact dermatitis in patients sensitized to topical metronidazole for the treatment of rosacea acne [4] or fixed drug eruptions [5].

In the present case, the patient did not have a family or personal history of allergy or contact dermatitis. Since we were not able to establish the timing of the reaction from the clinical history, we decided to perform both SPT and patch tests. Based on the different experiences described in the literature for patch tests, we used a metronidazole tablet (Flagyl) crushed and diluted initially at $0.5 \%, 5 \%$, and $10 \%$. Since these dilutions yielded negative results, we eventually applied the pure form. To exclude false positives, we performed the same test in 10 healthy controls; the results were negative. Most positive patch tests results with metronidazole in the literature were obtained from a fixed residual exanthema lesion. In the present case, we report another form of delayed systemic reaction, which was confirmed with a positive patch test result after administration of oral metronidazole.

The low sensitivity of SPTs and patch tests with metronidazole and their lack of standardization hamper diagnosis of metronidazole allergy. OPT is still essential for the diagnosis of early and delayed hypersensitivity reactions [1], even if it is not always feasible for ethical reasons. In a previous study [1], the authors presented 4 cases of cutaneous exanthema ( 2 early and 2 delayed). Only 1 patient had a positive metronidazole SPT result (all patch test results were negative). OPT with metronidazole was positive in the 3 patients with negative SPT results (delayed exanthema in the first patient and early erythema and itching in the other 2). According to these results, this test could be considered the gold standard for establishing a diagnosis of hypersensitivity reaction to metronidazole. In the present case, the patient denied previous treatment with oral or topical metronidazole; however, since the reaction was delayed, we hypothesize that he became sensitized during oral treatment. Furthermore, since cross-reactions with other imidazole derivatives have been reported in the literature, we cannot exclude indirect sensitization to metronidazole resulting from the use of other cross-reactive molecules. Imidazole derivatives are commonly found in many cosmetics, and it was difficult for the patient to remember using these substances in the past.

Cross-reactions have been reported between metronidazole and tinidazole [5] and between albendazole and metronidazole by oral challenge. Other authors did not observe reactivity between metronidazole, tinidazole, tioconazole, albendazole, ketoconazole, and mebendazole based on patch tests [2]. In our experience, the other imidazole derivatives do not seem to cross-react with metronidazole, although further studies should be performed in order to evaluate cross-reactivity in this drug group.

In conclusion, we report a rare delayed systemic reaction (angioedema and widespread erythematous maculopapular rash) to metronidazole after oral administration, with no apparent topical sensitization and confirmation by patch testing.

\section{Funding}

The authors declare that no funding was received for the present study.

\section{Conflicts of Interest}

The authors declare that they have no conflicts of interest.

\section{References}

1. García-Rubio I, Martínez-Cócera C, Santos Macadán S, Rodríguez-Jiménez B, Vázquez-Cortés S. Hypersensitivity reactions to metronidazole. Allergol Immunopathol (Madr). 2006;34(2):70-2.

2. Asensio Sánchez, Dávila I, Moreno E, Laffond E, Macías E, Ruiz $A$, et al. Anaphylaxis due to metronidazole with positive skin prick test. J Investig Allergol Clin Immunol. 2008;18(2):138-9.

3. Kurohara ML, Kwong FK, Lebherz TB, Klaustermeyer WB. Metronidazole hypersensitivity and oral desensitization. J Allergy Clin Immunol. 1991:88:279-80.

4. Fernández-Jorge $B$, Goday Buján J, Fernández-Torres $R$, Rodríguez-Lojo R, Fonseca E. Concomitant allergic contact dermatitis from diphenhydramine and metronidazole. Contact Dermatitis. 2008;59(2):115-6.

5. Prieto A, De Barrio M, Infante S, Torres A, Rubio M, Olalde S. Recurrent fixed drug eruption due to metronidazole elicited by patch test with tinidazole. Contact Dermatitis. 2005;53(3):169-70.

6. Aníbarro B, Fontela JL. Immediate rhinoconjunctivitis induced by metamizole and metronidazole. Ann Allergy Asthma Immunol. 1997;78:345-6.

7. Bedi RS. Metronidazole-induced asthma. Indian J Chest Dis Allied Sci. 1991;33(4):213-5.

8. Chen KT, Twu SJ, Chang HJ, Lin RS. Outbreak of StevensJohnson syndrome/toxic epidermal necrolysis associated with 
mebendazole and metronidazole use among Filipino laborers in Taiwan. Am J Public Health. 2003;93(3):489-92.

9. Watsky K. Acute generalized exanthematous pustulosis induced by metronidazole: the role of patch testing. Arch Dermatol. 1999;135:93-4.

10. Weart CW, Hyman LC. Serum sickness associated with metronidazole. South Med J. 1983;76(3):410-1.

- Manuscript received February 17, 2020; accepted for publication April 21, 2010.

Arianna Aruanno Allergy Unit, Fondazione Policlinico Universitario A. Gemelli IRCCS Largo F. Vito, 1 00168 Rome, Italy E-mail: arianna.aruanno@policlinicogemelli.it; aarianna@hotmail.it 